BRANCH AND TITHERLEY :

\title{
CCXLVII.-2-Phenyl-1:4:5:6-tetrahydropyrimidine and Benzoyl-ar-diaminopropane.
}

\section{By Grrald Eyre Kirkwood Branch and Arthur Walsh Titherley.}

In the synthesis of hexahydropyrimidine derivatives, to be described in a subsequent paper, it was desired to start with the monobenzoyl derivative of $\alpha \gamma$-diaminopropane,

$$
\mathrm{NH}_{2} \cdot \mathrm{CH}_{2} \cdot \mathrm{CH}_{2} \cdot \mathrm{CH}_{2} \cdot \mathrm{NHBz} \text {, }
$$

and submit it to the action of formaldehyde. Numerous unsuccessful attempts to obtain this compound, which has not been described, were made, and it is proposed to define these briefly, together with a final attempt which was successful, in which 2-phenyl-1:4:5:6-tetrahydropyrimidine was used. In the earlier attempts Gabriel and Weiner's a $\gamma$-diphthaliminopropane (Ber., $1888,21,2669$ ),

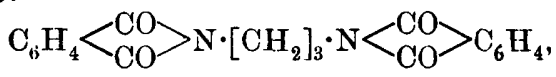

was used as a starting point, the intention being to submit the compound to half-hydrolysis and obtain monophthalyl-ay-diaminopropane. By benzoylation of this compound and subsequent elimination of the phthalyl residue, by alkali- and then acid-hydrolysis, monobenzoyl-ay-diaminopropane should be obtained. With this end in view the diphthalyl derivative was converted by Gabriel and Weiner's method (loc. cit.) into trimethylenediphthalamic acid, $\mathrm{CO}_{2} \mathrm{H} \cdot \mathrm{C}_{6} \mathrm{H}_{4} \cdot \mathrm{CO} \cdot \mathrm{NH} \cdot\left[\mathrm{CH}_{2}\right]_{3} \cdot \mathrm{NH} \cdot \mathrm{CO} \cdot \mathrm{C}_{6} \mathrm{H}_{4} \cdot \mathrm{CO}_{2} \mathrm{H}$.

The latter compound was found to be extremely sensitive to acidhydrolysis, but although many trials were made under varying conditions it was never found possible to eliminate phthalic acid in two stages. Even on heating with water ready hydrolysis occurs, but both phthalic groups are split off. The next attempt made was to eliminate one molecule of water and to obtain $\alpha$-phthaliminopropyl- $\gamma$-phthalamic acid,

$$
\mathrm{C}_{6} \mathrm{H}_{4}<\mathrm{CO}>\mathrm{N} \cdot\left[\mathrm{CH}_{2}\right]_{3} \cdot \mathrm{NH} \cdot \mathrm{CO} \cdot \mathrm{C}_{6} \mathrm{H}_{4} \cdot \mathrm{CO}_{2} \mathrm{H}
$$

with the object of submitting this to acid-hydrolysis, since it was found that the phthalimino-group, unlike the phthalamic residue, was very resistant to acid-hydrolysis, although sensitive to alkaline agents (with ring rupture only). Trimethylenediphthalamic acid was accordingly submitted cautiously to the influence of heat and dehydrating agents, but in all cases, even under very carefully moderated conditions, two molecules of water were eliminated, yielding the original diphthalimino-derivative, and this was true 
also when a comparatively small proportion of the total number of molecules were dehydrated. Conversely, attempts to prepare the desired monobasic acid by alkali-fission of one of the phthaliminorings, even under carefully moderated and very varied conditions, never yielded anything but the diphthalamic acid derivative produced by fission of both rings, and this was true also when the action had proceeded to a comparatively slight extent. It became obvious from the above negative results that if the attempted reactions, as may be supposed, take place in two successive stages, it is impossible even partly to restrict the action to the first stage, because the velocity of the second action must be greatly higher than that of the first. Attempts, which were next made, to prepare the compound

$$
\mathrm{C}_{6} \mathrm{H}_{4}<\mathrm{CO}>\mathrm{N} \cdot\left[\mathrm{CH}_{2}\right]_{3} \cdot \mathrm{NHBz}
$$

by the action of sodium benzamide on $\gamma$-bromopropylphthalimida proved equally unsuccessful.

The authors therefore turned next to the benzoylation of $a \gamma$-diaminopropane with the object of obtaining the monobenzoyl derivative, but here again the same difficulty referred to presented itself, and in spite of every precaution the dibenzoyl derivative, NHBz $\cdot\left[\mathrm{CH}_{2}\right]_{3} \cdot \mathrm{NHBz}$, described by Strache (Ber., 1888, 21, 2365) in a pure form always resulted. That is using $1 \mathrm{~mol}$. of benzoyl chloride or benzoic anhydride and $1 \mathrm{~mol}$. of a $\gamma$-diaminopropane, the $s$-dibenzoyl derivative results, and half of the diamine remains unaffected. This is probably to be explained in the case of the Schotten-Baumann reaction by the fact that since benzoylation takes place strictly in the oil (benzoyl chloride) and not in the aqueous phase, the concentration of the benzoyl chloride is at all moments of the reaction relatively very high. This explanation, however, cannot hold good in the case of other unsuccessful attempts made, when pyridine and acetone were used as solvents. Similarly, it was found impossible to obtain monobenzoyl- $\alpha$-diaminopropane by heating the monobenzoate of $a$-diaminopropane. Lastly an attempt was made to obtain it by treating the monoacetate of $a \gamma$-diaminopropane with benzaldehyde, benzoylating the resulting condensation derivative (after liberating the base from its acetate), and then cautiously hydrolysing to eliminate benzaldehyde. Nothing definite could be obtained in this way. The authors next turned to 2-phenyl-1:4:5:6-tetrahydropyrimidine (I) in the hope of effecting hydrolytic fission of the ring giving monobenzoyl$\alpha \gamma$-diaminopropane (II), thus :

$$
\mathrm{CPh} \leqslant \underset{\mathrm{N}-\mathrm{CH}_{2}}{\mathrm{NH} \cdot \mathrm{CH}_{2}}>\mathrm{CH}_{2} \rightarrow \mathrm{COPh} \cdot \mathrm{NH} \cdot \mathrm{CH}_{2} \cdot \mathrm{CH}_{2} \cdot \mathrm{CH}_{2} \cdot \mathrm{NH}_{2}
$$


2-Phenyl-1:4:5:6-tetrahydropyrimidine (I) has been already described in an impure form as an oil by Pinner (Ber., 1893, 26, 2122), who obtained it by the condensation of benzamidine and $a \gamma$-dibromopropane, which were allowed to react in ether-alcohol solution at $40^{\circ}$ for several weeks. The same compound is also mentioned by Hofmann (Ber., 1888, 21, 2337), who states that it is produced by heating dibenzoyl- $\alpha \gamma$-diaminopropane in hydrogen chloride, but he gives an unsatisfactory account of its isolation and properties. The authors have repeated Hofmann's work, and have failed to obtain the compound in this way, and it is evident that the substance described by Hofmann was obtained only in very small yield. It would appear that both Pinner's and Hofmann's products contained 2-phenyl-1:4:5:6-tetrahydropyrimidine, since each obtained correct values for -the analysis of the platinum double salt. Pinner, in the condensation referred to above. obtained as the main product of the reaction apparently bromopropylbenzamidine, $\mathrm{CPh}(: \mathrm{NH}) \cdot \mathrm{NH} \cdot\left[\mathrm{CH}_{2}\right]_{3} \cdot \mathrm{Br}$, which crystallised out incompletely on keeping, leaving an oil which could not be fractionated, but consisted largely of 2-phenyl-1:4:5:6-tetrahydropyrimidine, and this appears to be the purest form in which this compound has hitherto been obtained. The authors have cleared up the doubt concerning the compound by synthesising the pure substance by a modification of Pinner's method, acting on the following principles. The reaction between benzamidine and a dently takes place in successive stages, which may be represented thus:

$\mathrm{CPh} \leqslant \aleph_{\mathrm{NH}}^{\mathrm{NH}_{2}} \stackrel{\mathrm{Br}^{*}\left[\mathrm{CH}_{2}\right]_{3} \cdot \mathrm{Br}}{\longrightarrow} \quad \mathrm{CPh} \leqslant \underset{\mathrm{NH}}{\longrightarrow} \cdot\left[\mathrm{CH}_{2}\right]_{3} \cdot \mathrm{Br}, \mathrm{HBr} \quad \stackrel{\text { Benzamidine }}{\longrightarrow}$ Ammonium salt.

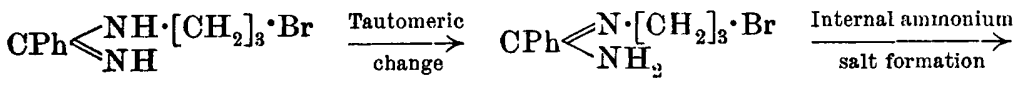
Free base. $\mathrm{CPh}<\underset{\mathrm{NH} \cdot \mathrm{CH}_{2}}{\mathrm{~N}-\mathrm{CH}_{2}}>\mathrm{CH}_{2}, \mathrm{HBr}$

The first stage is that of ordinary ammonium salt formation. The free base (incompletely liberated by the action of benzamidine present) can only undergo internal ammonium salt formation (leading to the tetrahydropyrimidine derivative) after tautomeric change. Except the latter, these reactions are of low (and unknown) velocity, which, however, would probably, like the ordinary Hofmann ammonium salt synthesis, be accelerated by alcohol. In view of these considerations it is clear that, in the synthesis of 2-phenyl-1:4:5:6-tetrahydropyrimidine, 2 mols. of benzamidine for 1 mol. of a $\gamma$-dibromopropane are necessary. With smaller quantities of benzamidine or insufficient time, the hydrobromide of $\omega$-bromopropylbenzamidine must remain. In practice these relative 
proportions were used in boiling alcohol for three days. It was found, however, that although the desired reaction was practically complete, the final yield of 2-phenyl-1:4:5:6-tetrahydropyrimidine was very small. This was partly due to secondary changes, such as the formation of allyl bromide, and slow decomposition of benzamidine by alcohol and traces of moisture which could not be excluded, giving ethyl benzoate and benzamide. Further, the extremely slow and laborious nature of the subsequent operations necessary to isolate the cyclic base tends to diminish the yield. The base is present in the reaction mixture, as its hydrobromide, together with benzamidine hydrobromide, and great difficulty was encountered in separating the two. 2-Phenyl-1:4:5:6-tetrahydropyrimidine when finally obtained in a pure condition is a crystalline solid, which becomes oily on treatment with water, and dissolves in excess. When heated with dilute ammonia at $80^{\circ}$ it slowly takes up water, and passes into monobenzoyl- $\alpha \gamma$-diaminopropane, probably on the following mechanism, which is similar to that described by one of the authors in the similar decomposition of 2-phenyl-1 : 3-benzoxazine-4-one (Titherley, Trans., 1910, 97, 201) :

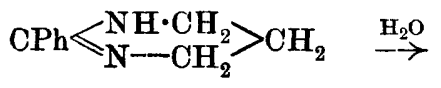

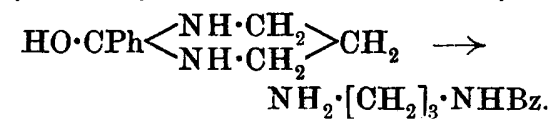

The resulting open-chain base, which is a crystalline solid readily soluble in water, may be isolated through its oxalate. Its constitu. tion follows from the fact that on hydrolysis it yields benzoic acid and $\alpha_{\gamma}$-diaminopropane, whilst on benzoylation it yields dibenzoyl$a \gamma$-diaminopropane, identical with that obtained directly from $a \gamma$-diaminopropane. The benzoylation of 2-phenyl-1:4:5:6-tetrahydropyrimidine by the Schotten-Baumann method gave unexpected results. Instead of the monobenzoyl derivative (III), tribenzoyl$a \gamma$-diaminopropane (IV) is produced, and, so far, the compound III has not been obtained. This anomalous reaction is readily intelligible if it is assumed that the monobenzoyl derivative (III), first formed, is very sensitive to water in presence of alkali (compare the behaviour of 2-phenyl-1:3-benzoxazine-4-one with acids); the intermediate compound (V) produced by addition of water being benzoylated, thus yields the compound VI, which is the cyclic tautomeric form of tribenzoyl- $\alpha \gamma$-diaminopropane (IV):
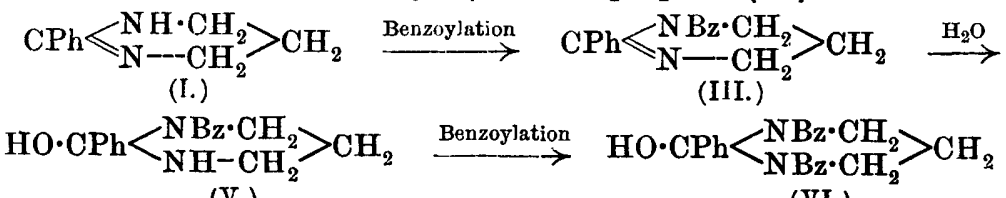

(V.)

$\rightarrow \mathrm{NBz}_{2} \cdot \mathrm{CH}_{2} \cdot \mathrm{CH}_{2} \cdot \mathrm{CH}_{2} \cdot \mathrm{NHBz}$

(VI.) 


\section{Experimental.}

The experiments $1-8$ described below refer to the attempts which were carried out with a view to the synthesis of benzoyl- $\alpha \gamma$ diaminopropane.

\section{(1) Hydrolysis of Trimethylenediphthalamic Acid.}

Acid hydrolysis, even when cautiously carried out, invariably gave phthalic acid and a $\gamma$-diaminopropane. By heating 0.5 gram of trimethylenediphthalamic acid with 5 c.c. of water at $100^{\circ}$ for four minutes, 20 per cent. was hydrolysed, yielding a $\gamma$-diaminopropane phthalate. The experiment was modified as to time, temperature, and concentration, but in no case was the desired $\gamma$-aminopropyl-a-phthalamic acid formed.

\section{(2) Dehydration of Trimethylenediphthalamic Acid.}

On heating at $100^{\circ}$ in the air, until the loss in weight corresponded with $1 \mathrm{~mol}$. of water, a semi-solid fusion was obtained, which, however, consisted of unchanged substance and diphthalyl$\alpha \gamma$-diaminopropane, melting at $198^{\circ}$.

The action of acetyl chloride, both free and in benzene solution, and of acetic anhydride in acetic acid solution, under various conditions gave only diphthalyl- $\alpha \gamma$-diaminopropane.

(3) Action of Alkali on Diphthalyl-ay-diaminopropane.

Half a gram of diphthalyl-ay-diaminopropane in 75 c.c. of alcohol at $80^{\circ}$ was treated cautiously (drop by drop) with one equivalent of $N / 10$-aqueous potassium hydroxide in the presence of phenolphthalein, so that the concentration of free alkali was always kept as low as possible. The alkali was fairly rapidly taken up, but rupture of both phthalimino-rings had occurred, giving the potassium salt of trimethylenediphthalamic acid, which after isolstion melted at $94^{\circ}$, and leaving nearly 0.25 gram of unchanged diphthalyl-ay-diaminopropane.

(4) Action of Benzoyl Chloride on Trimethylenediphthalamic Acid.

Several experiments were carried out at $60^{\circ}$ and at $120^{\circ}$ with different proportions of the reacting substances. Hydrogen chloride was given off, and diphthalyl- $\alpha \gamma$-diaminopropane (m. p. 198 $)$, together with $a \gamma$-diaminopropane hydrochloride, were found, but no benzoylphthalyl-a $\gamma$-diaminopropane. 
(5) Action of Sodium Benzamide on $\gamma$-Bromopropylphthalimide.

Equivalent quantities were heated at $125-130^{\circ}$ for an hour. Free benzamide and sodium bromide were formed in quantity, together apparently with phthalylallylamine, which, however, was not investigated. No benzoylphthalyl- $a \gamma$-diaminopropane was formed.

\section{(6) Benzoylation of a $\gamma$-Diaminopropane.}

By the Schotten-Baumann method, using one equivalent of benzoyl chloride, only half the diamine reacted, giving the dibenzoyl derivative (m. p. $151^{\circ}$ ). The same compound resulted by benzoylation in pyridine solution with benzoic anhydride, also by benzoylation of $a \gamma$-diaminopropane monobenzoate or monohydrochloride in acetone solution with benzoic anhydride. In all cases, using one equivalent of benzoic anhydride, half the diamine remained unaffected, and the remainder gave the dibenzoyl derivative.

\section{(7) Action of Heat on a $\gamma$-Diaminopropane Monobenzoate.}

At $170-180^{\circ}$ for six hours darkening occurred, but no monobenzoyl-a $\gamma$-diaminopropane could be isolated from the product.

(8) Condensation of a $\gamma$-Diaminopropane Monoacetate and Benzaldehyde.

The reaction was carried out in alcoholic solution, and a discoloured, viscid syrup was obtained after evaporation. This syrup, which is probably the impure acetate of benzylidene- $\alpha \gamma$-diaminopropane, has not been further investigated, but attempts to benzoylate it in pyridine and subsequently eliminate benzaldehyde by acid hydrolysis, in order to obtain monobenzoyl-a $\gamma$-diaminopropane, were unsuccessful.

\section{Condensation of Benzamidine and a -Dibromopropane.}

After several trials the following method gave the best results:

Forty-five grams of benzamidine hydrochloride in the minimum quantity of water were treated at $0^{\circ}$ with the equivalent amount of 50 per cent. aqueous potassium hydroxide. The solution was then made into a thin paste with an excess of anhydrous potassium carbonate, and the free benzamidine extracted several times with alcohol (about 200 c.c.). The alcoholic solution, after drying with 
anhydrous potassium carbonate, was heated to boiling in a reflux apparatus with 23.4 grams of a -dibromopropane for three days.

The alcohol was removed from the product at the ordinary temperature in a vacuum, and the semi-solid mass remaining, consisting essentially of the hydrobromides of benzamidine and 2-phenyl-1:4:5:6-tetrahydropyrimidine, was treated with water and extracted with ether. The ethereal extrace yielded about 2.5 grams of a mixture of benzamide and ethyl benzoate.

The aqueous solution was then treated in the cold with excess of 50 per cent. aqueous potassium hydroxide, and the yellow oil produced was extracted with benzene. The benzene was removed in a vacuum, and the mixed bases remaining were neutralised exactly (using litmus) with aqueous oxalic acid. The aqueous solution after filtering from benzamide ( 1.8 grams) was concentrated in the cold over sulphuric acid in a vacuum. The residual mass of syrup and crystals (normal oxalates) weighing 35 grams was extracted with alcohol, and the insoluble benzamidine oxalate collected. The alcohol filtrate was again evaporated, and, as before, the residue once more extracted with alcohol, and the insoluble benzamidine oxalate separated. In this way most of the benzamidine was removed as insoluble oxalate (total weight 20.6 grams), whilst the oxalate of the cyclic base remained in solution. The latter on evaporation remained as a syrup, which crystallised only with difficulty. It was therefore converted into the hydrogen oxalate by treatment with an excess of anhydrous oxalic acid in acetone solution, when the hydrogen oxalate of the cyclic base separated as a white, crystalline solid ( 5.7 grams). The mother liquor still contained small quantities in solution, which were not recovered, together with benzamidine oxalate, and these were precipitated in syrupy form on adding more acetone. This syrup on decomposition with hot dilute aqueous ammonia gave 0.5 gram of benzamide and 0.6 gram of monobenzoyl-a $\gamma$-diaminopropane (p. 2350), which was isolated as its oxalate.

$$
\begin{aligned}
& \text { 2-Phenyl-1:4:5:6-tetrahydropyrimidine, } \\
& \mathrm{CPh} \leqslant \mathrm{NH} \cdot \mathrm{CH}_{2}>\mathrm{CH}_{2}>\mathrm{CH}_{2} .
\end{aligned}
$$

The free base was isolated from the hydrogen oxalate, described above, by treating its saturated solution in water with 50 per cent. aqueous potassium hydroxide and extracting the oil with a small quantity of ether. The dried ethereal solution was deprived of ether at $20^{\circ}$ by a current of pure dry air, when the base remained as a colourless oil, which rapidly set to a solid, white, crystalline mass melting at $72-78^{\circ}$ : 
$0 \cdot 1433$, by Kjeldahl's method, required $18 \cdot 0$ c.c. $N / 10-\mathrm{HCl}$. $\mathrm{N}=17 \cdot 58$.

$$
\mathrm{C}_{10} \mathrm{H}_{12} \mathrm{~N}_{2} \text { requires } \mathrm{N}=17 \cdot 50 \text { per cent. }
$$

2-Phenyl-1:4:5:6-tetrahydropyrimidine is readily soluble in ether or benzene, and excessively so in alcohol. With a small quantity of water it changes to an oil, which dissolves in about fifty parts of water, giving a strongly alkaline solution, which on spontaneous evaporation in air leaves a crystalline carbonate. On heating with dilute aqueous ammonia, the cyclic base slowly passes into monobenzoyl-a $\gamma$-diaminopropane (see p. 2350).

The hydrochloride crystallises slowly in long, colourless prisms from the syrup obtained on allowing its aqueous solution to evaporate in the air.

The nitrate crystallises readily on evaporating its aqueous solution.

The picrate separates from aqueous solutions as a microcrystalline, yellow precipitate.

The platinichloride separates from an aqueous solution as a microcrystalline powder, which melts and decomposes at $213-214^{\circ}$. For analysis it was dried at $100^{\circ}$ :

0.0879 gave $0.0233 \mathrm{Pt}$. $\mathrm{Pt}=26.52$.

$\left(\mathrm{C}_{10} \mathrm{H}_{12} \mathrm{~N}_{2}, \mathrm{HCl}\right)_{2} \mathrm{PtCl}_{4}$ requires $\mathrm{Pt}=26.66$ per cent.

The oxalate on evaporating its aqueous solution prepared from the base, remains as a syrup, which slowly crystallises in a vacuum and melts at $175^{\circ}$. It is readily soluble in alcohol, and excessively soluble in water.

The hydrogen oxalate (described above) melts at $180^{\circ}$, and is very soluble in water, and moderately so in alcohol.

Benzoylation of 2-Phenyl-1:4:5:6-tetrahydropyrimidine:

Tribenzoyl-ay-diaminopropane, $\mathrm{NBz}_{2} \cdot \mathrm{CH}_{2} \cdot \mathrm{CH}_{2} \cdot \mathrm{CH}_{2} \cdot \mathrm{NHBz}$.

By the Schotten-Baumann method the pyrimidine base is readily benzoylated, but takes up two molecules of benzoyl chloride, and the ring is broken. An oil was obtained, which solidified on keeping. After recrystallisation from benzene and light petroleum, and finally from aqueous alcohol, colourless needles were obtained melting at $144^{\circ}$ :

$0 \cdot 1980$, by Kjeldahl's method, required $10 \cdot 6$ c.c. $N / 10-\mathrm{HCl}$. $\mathrm{N}=7 \cdot 49$.

0.0776 , by Kjeldahl's method, required 4.0 c.c. $N / 10-\mathrm{HCl}$. $\mathrm{N}=7 \cdot 22$.

$$
\mathrm{C}_{24} \mathrm{H}_{22} \mathrm{O}_{3} \mathrm{~N}_{2} \text { requires } \mathrm{N}=7 \cdot 25 \text { per cent. }
$$

The constitution of the compound follows from the fact that on 
cautious hydrolysis with 10 per cent. aqueous alkali at $100^{\circ}$ for thirty-five minutes it yields benzoic acid and dibenzoyl-ay-diaminopropane. The latter melted at $150^{\circ}$, and a mixture with a pure specimen obtained from $a \gamma$-diaminopropane melted at $150^{\circ}$. Attempts to confirm the fact that the compound melting at $144^{\circ}$ is tribenzoyl-a $\gamma$-diaminopropane by pyridine benzoylation of dibenzoyl- $\alpha \gamma$-diaminopropane failed, because the latter, probably from steric causes, was not benzoylated even after eight days, and the unchanged material was entirely recovered. Tribenzoyl-a $\gamma$-diaminopropane is soluble in cold alcohol, although less so than the dibenzoyl derivative. It is readily soluble in benzene, but sparingly so in ether.

\section{Monobenzoyl-ay-diaminopropane, $\mathrm{NH}_{2} \cdot \mathrm{CH}_{2} \cdot \mathrm{CH}_{2} \cdot \mathrm{CH}_{2} \cdot \mathrm{NHBz}$.}

In the synthesis of this base, through 2-phenyl-1:4:5:6-tetrahydropyrimidine, the actual isolation of the latter was not found to be necessary, and the following treatment was adopted:

An alcoholic solution of benzamidine from 10 grams of the hydrochloride was heated as described above with 5.2 grams of $\alpha \gamma$-dibromopropane for three days. The alcohol was removed from the product at the ordinary temperature in a vacuum, the syrupy residue consisting chiefly of the hydrobromides of 2-phenyl$1: 4: 5: 6$-tetrahydropyrimidine and benzamidine, was treated with a little water and freed from impurities (benzamide and ethyl benzoate) by extracting three times with ether. The aqueous solution was then treated with excess of 50 per cent. potassium hydroxide, and the oily mixed bases were extracted twice with benzene. After remaining over solid potassium hydroxide, the benzene was removed at the ordinary temperature in a vacuum, leaving a brown oil ( 8.3 grams). In order to remove as much benzamidine as possible the oil was dissolved in 25 c.c. of alcohol and saturated with moist carbon dioxide. After filtering from the precipitated benzamidine carbonate $(2.5$ grams) and evaporating in a vacuum, a mixture of oil and crystals was left (6.7 grams). The mixture was treated with 30 c.c. of water and 5 c.c. of concentrated aqueous ammonia, and heated in a reflux apparatus at $80^{\circ}$. The oil gradually disappeared, and after three hours the clear solution was evaporated to small bulk on the water-bath. After being kept in the cold, crystals of benzamide (1.46 grams) which separated were removed, and the filtrate was just neutralised with 10 per cent. hydrochloric acid. The last traces of benzamide were now removed by repeated extraction with ether, the aqueous solution was then treated with excess of 50 per cent. potassium 
hydroxide, and the oily base thus salted out was extracted twice with ethyl acetate. After removing the solvent in a vacuum, the crude base was left as a slightly discoloured oil (2.7 grams), which was purified by conversion into its normal oxalate by treatment with an alcoholic solution of anhydrous oxalic acid. The oxalate was obtained as a white, crystalline precipitate $(2.0$ grams), which was purified by recrystallisation from boiling methyl alcohol.

Isotation of the Free Base.-A concentrated aqueous solution of the oxalate was treated with 50 per cent. potassium hydroxide in excess, and the oil extracted by a large quantity of pure ether, in which it is not very soluble. The dried ethereal solution was freed from ether at $30^{\circ}$ by means of a current of air, free from carbon dioxide and moisture. The base remained as a very pale yellow liquid, which on keeping crystallised in stout, colourless, transparent prisms, melting at $46^{\circ}$ :

$0 \cdot 1885$, by Kjeldahl's method, required $20 \cdot 6$ c.c. $N / 10-\mathrm{HCl}$. $\mathrm{N}=15 \cdot 3$.

$$
\mathrm{C}_{10} \mathrm{H}_{14} \mathrm{ON}_{2} \text { requires } \mathrm{N}=15 \cdot 7 \text { per cent. }
$$

Monobenzoyl-ay-diaminopropane is very soluble in water or alcohol, readily so in chloroform or benzene, but only moderately so in ether (about 1 in 40). The following salts have been prepared:

The Carbonate.-An aqueous solution of the base absorbs carbon dioxide from the air, and on keeping leaves a crystalline carbonate. The same salt, which is readily soluble in water, but only moderately so in alcohol, was prepared from the base dissolved in alcohol by moist carbon dioxide:

0.2183 required 10.25 c.c. $N / 10-\mathrm{HCl}$. This corresponds with the formula $2 \mathrm{C}_{10} \mathrm{H}_{14} \mathrm{ON}_{2}, \mathrm{H}_{2} \mathrm{CO}_{3}$.

The hydrochloride, on evaporating its aqueous solution in a vacuum, remains as a syrup which crystallises (in tablets) only after a long time.

The platinichloride separates from fairly concentrated aqueous solutions as a yellow, microcrystalline precipitate; after recrystallisation from hot water it forms irregular, orange crystals, soluble in boiling alcohol, and melting and decomposing at $194^{\circ}$. For analysis it was dried at $100^{\circ}$ :

0.1714 gave $0.0436 \mathrm{Pt}$. $\mathrm{Pt}=25.44$.

$\left(\mathrm{C}_{10} \mathrm{H}_{14} \mathrm{ON}_{2}, \mathrm{HCl}\right)_{2} \mathrm{PtCl}_{4}$ requires $\mathrm{Pt}=25 \cdot 46$ per cent.

The picrate separates from aqueous solutions of the base as a brownish-yellow, oily precipitate.

The oxalate, described above, crystallises in colourless needles, and melts and decomposes at $196^{\circ}$; it is readily soluble in water, but practically insoluble in alcohol:

VOL. CI. 
$0 \cdot 1870$, by Kjeldahl's method, required $17 \cdot 0$ c.c. $N / 10-$ HCl. $\mathrm{N}=12 \cdot 7$.

$$
2 \mathrm{C}_{10} \mathrm{H}_{14} \mathrm{ON}_{2}, \mathrm{H}_{2} \mathrm{C}_{2} \mathrm{O}_{4} \text { requires } \mathrm{N}=12 \cdot 6 \text { per cent. }
$$

The hydrogen oxalate was obtained by treating the normal salt with an excess of oxalic acid in aqueous solution, evaporating, and extracting with ether. It is a colourless, crystalline solid, readily soluble in alcohol (compare the normal salt). When the dilute alcoholic solution is precipitated by cautious addition of ether, a mass of needles is obtained, consisting of a mixture of normal and hydrogen oxalates of the base, owing to partial dissociation.

\section{Hydrolysis of Monobenzoyl-ay-diaminopropane.}

By heating at $90^{\circ}$ with 10 per cent. aqueous hydrochloric acid for seventy-five minutes the compound was completely hydrolysed, yielding benzoic acid and a $\gamma$-diaminopropane hydrochloride. By heating at $90^{\circ}$ with 10 per cent. potassium hydroxide for two and a-quarter hours hydrolysis occurred to the extent of 90 per cent.

Benzoylation of Monobenzoyl-ay-diaminopropane.

The base is very readily benzoylated by the Schotten-Baumann method, giving a practically quantitative yield of dibenzoyl- $\alpha \gamma-$ diaminopropane (m. p. $151^{\circ}$ ). (Found, $\mathrm{N}=10.04$. Calc., $\mathrm{N}=9.93$ per cent.)

A mixture of the substance with pure dibenzoyl-a $\gamma$-diaminopropane, obtained from a $\gamma$-diaminopropane, melted at $151^{\circ}$.

Organic laboratory, UNIVERSITY OF LIVEKPOOL. 